\title{
ON THE TOTAL LOSS PROBABILITY OF SERVICE STAGES IN SERIES
}

\author{
P.-J. COURTOIS, ${ }^{*}$ Philips Research Laboratory, Brussels
}

\begin{abstract}
The problem addressed here is related to the minimization of the total loss probability in series of finite queues at which customers are rejected if the waiting capacity is exceeded. More precisely, one is concerned with the question of determining whether or not there may exist conditions under which an increase of the loss rate at one queue, e.g. at the most upstream one, could result in a decrease of the total loss rate throughout the whole network. The answer obtained in the context of a simple model is negative.
\end{abstract}

TANDEM QUEUES; LOSS PROBABILITY

\section{Introduction}

The problem analysed here originated in the course of a study of optimal sizes for finite waiting queues being used in series. All queues were supposed to be loss queues: a customer who completes service at one queue joins the next one if it is not full, and is rejected from the network otherwise. The queues were to be designed so as to minimize the total rejection probability at any queue of the serial network.

The question was to know whether, under certain conditions, an increase of the rejection rate at one queue, for instance at the most upstream one, could be compensated by a decrease of the rejection rate at the downstream queues, and could result in a decrease of the total rejection rate throughout the network. Or, more precisely, whether there exist associations of interarrival time and service time distributions such that the loss of a customer at a queue could, on the average, avoid the loss of more than one customer at any downstream queue.

These questions are answered here for a simple model of two finite-capacity service stages in tandem, with independent Bernoulli processes for arrivals and for services and with no queueing. The answers to both questions are negative, although the assumptions of the model are biased towards the opposite. How these results should be interpreted when queueing is introduced is briefly discussed at the end.

\section{Two finite-capacity service stages in tandem}

Suppose a system of two finite service stages in tandem which satisfies the following assumptions:

1. The arrival process at stage 1 , and the service processes at stage 1 and 2 are all independent from each other.

2. Every new customer arriving at stage 1 is rejected, or accepted, with fixed probability $y$ or $(1-y)$, respectively, $0 \leqq y \leqq 1$.

3. With respect to stage 2, customers are of one of two types. Customers of type 1 cause no subsequent loss when they join stage 2 . Customers of type 2 , if they join stage 2 , cause the loss

Received 31 August 1988; revision received 1 September 1989.

* Postal address: Philips Research Laboratory, Av. E. Van Becelaere 2, Box 8, B-1170 Brussels, Belgium. 
of the $n$ subsequent customers who complete service at stage 1 . A customer is of the first, or second type, with fixed probability $(1-z)$, or $z$, respectively, $0 \leqq z \leqq 1$.

4. This loss of $n$ customers is independent from $y$; we shall see later that our results are insensitive to this assumption.

5. Probabilities $y$ and $z$ are independent from each other; $y z$ is therefore the probability of rejecting at stage 1 a customer who, upon joining stage 2, would cause $n$ losses at this stage.

Call $P(y, z)$ the probability of a customer being lost at either stage 1 or stage 2 . When $y$ is increased-for instance by decreasing the size of stage 1-the number of customers of type 2 who will join and cause losses at stage 2 will be reduced. How, as a result of this reduction, is $P(y, z)$ varying in function of $y$ ? The following lemma gives an answer.

Lemma 1 . For any $y, z, n, 0 \leqq y, z \leqq 1, n \in \mathbb{N}$,

$$
P(y, z)=y+(1-y) \frac{n z}{1+n z},
$$

and is therefore a non-decreasing function of $y$.

Proof. The probability of a loss at stage 1 is $y$. At stage $2, n$ customers are lost if a type 2 customer joins stage 2 . Let $Q$ be the probability of this last event. Thus $n Q$ is the probability that a customer completing service at stage 1 is rejected at stage 2 . To compute $Q$, let $\pi(1)$ be the probability that stage 2 is occupied by a customer of type 1 , and let $\pi_{n-k}^{(2)}, k=0, \cdots, n$, be the probability that stage 2 is occupied by a customer of type 2 and that $k$ subsequent customers completing service at stage 1 have already been rejected. We have:

$$
\pi^{(1)}+\sum_{k=0}^{n} \pi_{n-k}^{(2)}=1, \quad \text { and } Q=\pi_{n}^{(2)}=\pi_{n-1}^{(2)}=\cdots=\pi_{0}^{(2)} .
$$

A customer can join stage 2 if the last one to join was of type 1 or if it were of type 2 and $n$ customers have been rejected. Thus, a customer of type 2 joins stage 2 with probability:

thus

$$
\pi_{n}^{(2)}=z\left(\pi^{(1)}+\pi_{0}^{(2)}\right)
$$

so that

$$
\pi_{n}^{(2)}(1-z)=\pi^{(1)} z=z\left[1-(n+1) \pi_{2}^{(2)}\right]
$$

which completes the proof.

$$
Q=\pi_{n}^{(2)}=\frac{z}{1+n z}
$$

A second 'zero gain per loss' principle can be established. Whenever a type 2 customer-who would have joined stage 2 -is rejected at stage 1 (with probability $y$ ), a certain number of customers are 'saved' from rejection at stage 2 . We shall prove that the relative number of customers who can be saved in this way is always smaller than the relative number of customers of any type rejected at the first stage.

More precisely, if $G(y, z)$ is the probability of a customer being saved from rejection at stage 2 , then the following result holds.

Lemma 2. For any $y, z(0 \leqq y, z \leqq 1)$, and $n, n \in \mathbb{N}$ :

$$
G(y, z)=\frac{y}{1+n z}\left(1-(1-z)^{n}\right) \leqq y .
$$

Proof. Whenever a type 2 customer is rejected at stage $1,(k+1)$ customers are saved from rejection at stage 2 , if $k, k \leqq n-1$, is the number of consecutive type 1 customers who follow this type 2 customer. Let $\bar{S}$ be the mean of this number $(k+1)$ of saved customers; this number can be computed over the probabilities of having $0,1,2, \cdots, n$ customers of type 2 among $n$ customers, each time taking into account all the possible positions that may be 
occupied by the first of these customers:

$$
\begin{aligned}
\bar{S}=n(1-z)^{n}+ & z(1-z)^{n-1}(1+2+\cdots n)+\cdots+z^{k}(1-z)^{n-k} \sum_{i=1}^{n-k+1} i\left(\begin{array}{c}
n-i \\
k-1
\end{array}\right)+\cdots+z^{n} \\
\bar{S} & =n(1-z)^{n}+\sum_{k=1}^{n} z^{k}(1-z)^{n-k} \sum_{i=1}^{n-k+1} i\left(\begin{array}{c}
n-i \\
k-1
\end{array}\right) \\
& =n(1-z)^{n}+\sum_{i=1}^{n} i \sum_{k=1}^{n}\left(\begin{array}{c}
n-i \\
k-1
\end{array}\right) z^{k}(1-z)^{n-k} \\
& =n(1-z)^{n}+z \sum_{i=1}^{n} i(1-z)^{i-1} \\
& =n(1-z)^{n}+\frac{1-(1-z)^{n}-n(1-z)^{n} z}{z}=z^{-1}\left(1-(1-z)^{n}\right) .
\end{aligned}
$$

The probability of a customer being saved by a type 2 customer rejection at stage 1 is equal to $y z(1-Q) \bar{S}$, where $Q$, the probability of a customer being lost at stage 2 , is given in the proof of Lemma 1. This completes the proof.

It is easy to see that these results are insensitive to Assumption 4. Both lemmas remain true independently of whether $n$ is an increasing or decreasing function of $y$. It is also easy to conceive queueing models which obey the five assumptions; for instance, queues with Bernoulli arrivals and services; the latter are at the second queue of one of two types, very long or very short.

\section{Acknowledgements}

The problem discussed here was first formulated in discussions with G. Scheys and P. Semal, Philips Research Laboratory. The author also thanks his colleague Ph. Delsarte for his help in the computation of $\bar{S}$. 